\title{
A Portable Measuring Instrument for Assessing Physical Activity Based on Triaxial Accelerometer
}

\author{
Chun Jiao, Xuyang Huo, Ming Tang \\ School of Intelligence Science and Information Engineering \\ Xi'an Peihua University, Xi'an, China \\ 2024988445@qq.com
}

\section{Keywords: Assessing physical activity; Energy expenditure; Triaxial accelerometer}

\begin{abstract}
The usefulness of accelerometer for assessing physical activity has been widely accepted in recent years. In this paper, a portable measuring instrument based on triaxial accelerometer was presented, also the overall design scheme of the portable measuring instrument was expounded emphatically, and combined with the principles of low-power design and miniaturization design, the realization method of system design, signal conditioning unit and software design in the whole system was illustrated in detail.
\end{abstract}

\section{Introduction}

Physical activity is very important to maintain the health of human body, which is helpful to prevent obesity, diabetes, hyperlipidemia, cardiovascular disease etc. In another way, superfluous exercise is harmful to our body, which would lead to acute or chronic athletic injury, sometimes even cause sudden death. Therefore moderate physical activity is preferred for human body, in the case of which the reliable assessment of the intensity or the amount of ambulatory movements in daily life is a determining factor. In recent years, the amount of energy consumption due to daily physical activity has been widely accepted as an objective basis for evaluating physical activity.

The studies from Bouten, Troiano and Klippel have verified that the output of accelerometer is highly correlated with energy expenditure of physical activity [1][2][3], which provides theoretical basis for assessing energy expenditure of physical activity by accelerometer. Moreover, more and more studies have confirmed the reliability and validity when physical activity is assessed by triaxial accelerometer [4][5][6][7]. In 2011, Daniel analyzed the study results of one thousand nine hundred and twenty-four articles, then summarized that the measuring method for assessing energy expenditure of physical activity based on accelerometer had thoroughly changed the research on assessing physical activity [8].

In this paper, a portable measuring instrument for assessing physical activity based on triaxial accelerometer was expounded, which was able to save sports data and calculate estimated value of energy expenditure of physical activity in daily life.

\section{The System Design}

The portable measuring instrument for assessing physical activity based on triaxial accelerometer was a portable application system of single-chip controller that was battery-powered, which consisted of hardware units and software units. The portable measuring instrument was able to amplify, shape, and filter motion acceleration signal, then collect processed signal and save all data, an estimated value of energy expenditure of physical activity was calculated by specific algorithm, which would give guidance to daily exercise, diet structure or health promotion program.

The structure diagram of the portable measuring instrument for assessing physical activity based on triaxial accelerometer is shown in Fig.1.

In the measuring instrument, a combined three-dimensional acceleration sensor was used to assess various movement of the body which was made up of three uniaxial acceleration sensors(A1,A2 and A3) that were mounted orthogonally onto the circuit board of the instrument. A1, A2 and A3 were uniaxial piezoresistive acceleration sensors which were able to measure antero-posterior, vertical and 
medio-lateral acceleration severally. The acceleration sensors should be zeroed at their quiescent state by compensation circuit of zero offset because each acceleration sensor had its own zero acceleration output which would affect the sensitivity of the instrument.

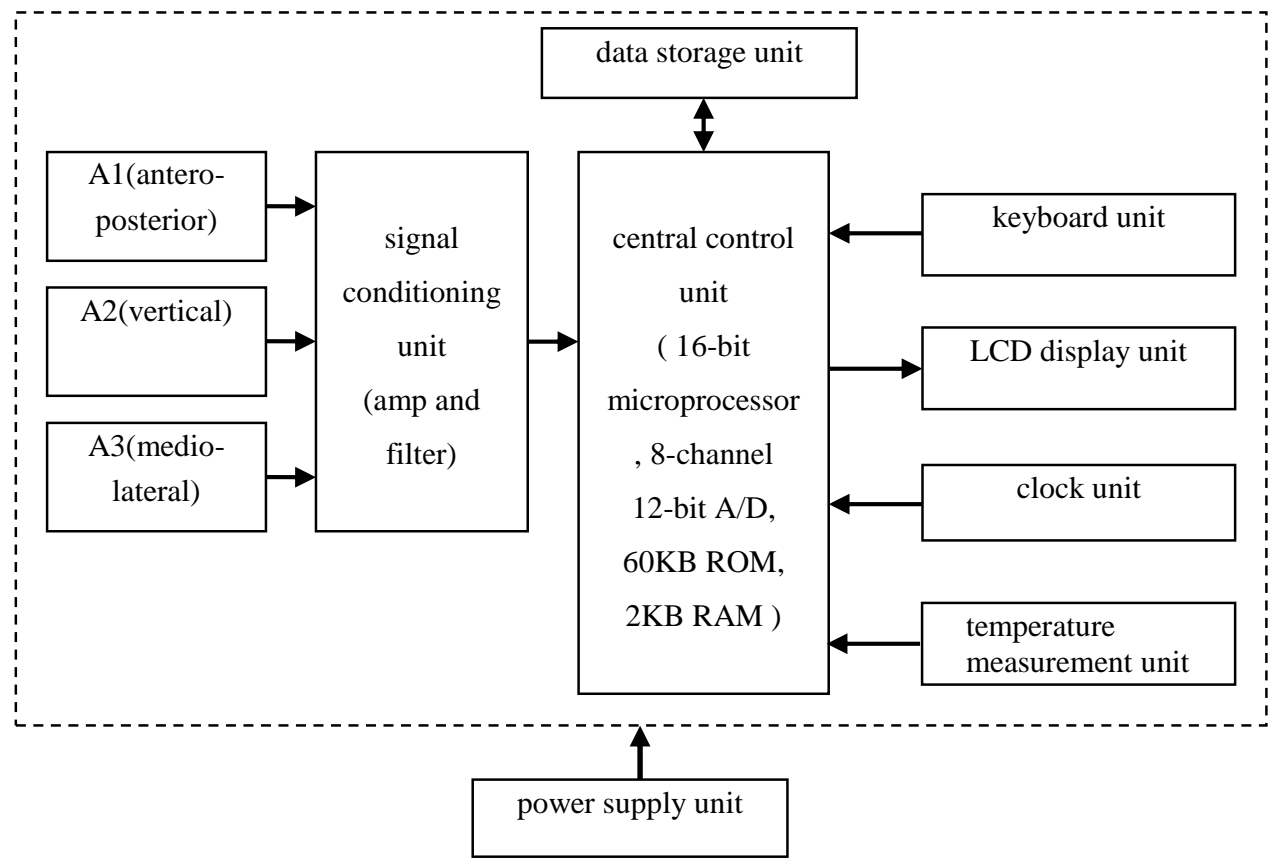

Figure 1. The structure diagram of the portable measuring instrument

For assessing physical activity in daily life, the portable measuring instrument should be put at waist level of the body since a lot of research has suggested. During locomotion, the amplitudes of accelerations at waist level of the body usually range from $-6 \mathrm{~g}$ to $+6 \mathrm{~g}$, and the frequencies of accelerations are up to $20 \mathrm{~Hz}$. As shown in Fig.1, the signal conditioning unit consisted of amplifier, filter and voltage reference circuit. Sports signals from three acceleration sensors passed through the signal conditioning unit, which would amplify, shape and filter the signals accurately to correct form and amplitude that was able to meet the requirements of A/D module. As shown in Fig.2, the portable measuring instrument was attached on the waist of human body.

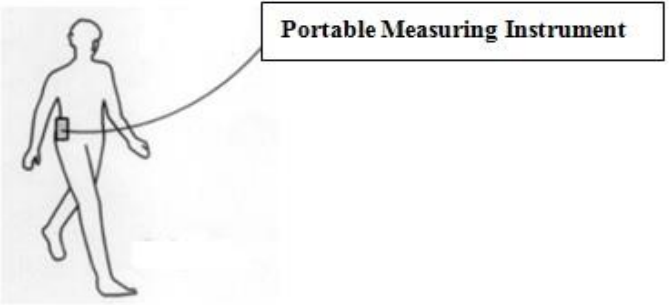

Figure 2. The attached location of the portable measuring instrument on the body

As the core of the whole system, the central control unit was very important for the design of whole system, especially for the control on power consumption. As a 16-bit microprocessor, MSP430F149 was selected as the microcontroller of central control unit, which has characteristics of high integration and ultralow power consumption, and contains $60 \mathrm{~KB}$ flash rom, $2 \mathrm{~KB}$ data ram, 8-channel A/D converter with 12-bit precision, hardware multiplier, $\mathrm{I}^{2} \mathrm{C}$ port, SPI port, UART port and so on. With its internal A/D converter which has high precision and fast conversion speed, no extra A/D circuit was needed in the system, which reduced size of the system. It has special low-power modes for ultralow-power applications, which is able to reduce its own power consumption and minimize individual peripheral current consumption. At the same time, a specific algorithm for assessing energy expenditure of physical activity was carried out by the internal hardware multiplier of MSP430F149.

The data storage unit was used to store sampled acceleration data for processing subsequently. Referring to the choice of memory chip, factors in all aspects such as power consumption, storage capacity, access speed, interface form and circuit volume should be considered in an integrated 
manner. AT45DB321B was selected as the main chip of data storage unit, which has a large storage capacity of $4 \mathrm{M}$ bytes and is low power consumption with low voltage power supply, $2 \mu \mathrm{A}$ standby current, $4 \mathrm{~mA}$ read current and $15 \mathrm{~mA}$ burning current consumption. AT45DB321B was connected with MSP430F149 by SPI port through five pins. As shown in Fig.3, UCLK0 pin, SIMO0 pin and SOMI0 pin of MSP430F149 were connected with SCK pin, SI pin and SO pin of AT45DB321B, which were used to exchange data with each other. P3.4 pin of MSP430F149 was used to enable AT45DB321B, P1.7 pin of MSP430F149 was used to get status information from AT45DB321B. And for filtering out the noise to avoid misoperation, $100 \mathrm{pF}$ decoupling capacitors were connected with SCK pin, SI pin and CS pin of AT45DB321B. AT45DB321B contains two 528-byte data buffers that enables it to receive data even when it is written, which greatly improves reliability. A page program in AT45DB321B would be started that 528 bytes would be saved one time. Compared with regular page with 512 bytes, a page with 528 bytes in AT45DB321B provides extra 16 bytes to detect errors or store specific control information, such as address pointer, check code, specific flags and so on. The connection diagram between AT45DB321B and MSP430F149 is shown in Fig.3.

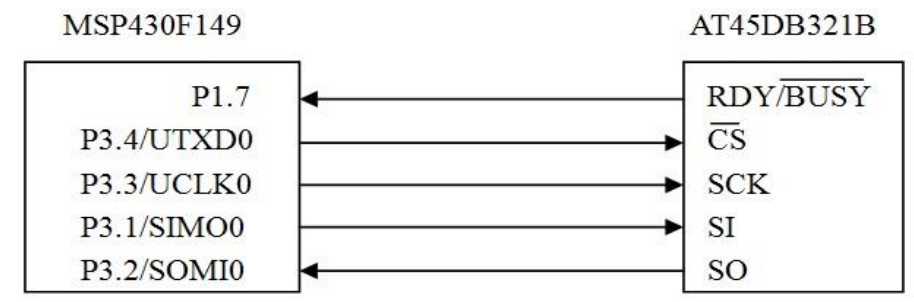

Figure 3. The connection diagram between AT45DB321B and MSP430F149

The temperature measurement unit was used to provide environment temperature for the system, which would offer a temperature reference for the assessed value of energy expenditure of physical activity. TMP100 was selected as the main chip of the unit, which has outstanding advantages in low power consumption with $45 \mu \mathrm{A}$ quiescent current and $0.1 \mu \mathrm{A}$ standby current. TMP100 is very small with SOT23-6 package. In temperature measurement program, one-shot measuring mode was carried out to start a temperature measurement, which was able to return to cut-off mode after conversion.

The clock unit was used to provide a time reference for recording sports signals in the whole system. Combined with sports classification algorithm, different types and duration of exercise would be recorded, and it was able to calculate energy consumption of ambulatory movements in a specific time period by the time scale offered by the unit.

The keyboard unit and the LCD display unit were used to serve as a simple and convenient man-machine interactive interface for users. The keyboard unit was able to provide an interface to setup and operate the instrument. The LCD display unit was able to display Chinese character menu, prompt messages and status information, and would display waveform of sports signals when necessary.

\section{The Signal Conditioning Unit}

Output signals from three piezoresistive acceleration sensors are of millivolt level, which should be converted to voltage signal with suitable amplitude for sampling by A/D module of MSP430F149. The signal conditioning unit included amplification circuit, low-pass filter circuit and voltage reference circuit.

The low-pass filter circuit was used to filter out signals higher than $20 \mathrm{~Hz}$ that can not be expected to arise from body movements. In the design of filter circuit, a second order active butterworth filter was carried out, which was made up of an in-phase operational amplifier with high input impedance and $\mathrm{RC}$ network. And there was no ripple in passband of the filter.

The voltage reference circuit was used to provide a $1.25 \mathrm{~V}$ voltage reference since the A/D module of MSP430F149 has an voltage interval that is from $0 \mathrm{~V}$ to $2.5 \mathrm{~V}$.

In the design of signal conditioning unit, the amplification circuit was key point, which should have high common-mode rejection ratio, high input impedance, low temperature drift and low offset 
voltage, also it should have low power consumption. An in-phase instrumentation amplifier with differential structure was carried out that had the characteristics of wide gain adjustable range.

The gain range of amplification circuit was able to be quantificationally calculated by the following formula.

$$
\text { gain range }=\frac{\text { full scale voltage range of amplified signal }}{\text { voltage range of measured signal } \times \text { sensor's sensitivity } \times \text { passband gain of filter }}
$$

\section{The software design in the system}

As shown in Fig.4, the software of measuring instrument was composed of main control program and several function programs, such as A/D conversion program, data read/write program, motion energy expenditure calculation program, LCD display program, keyboard input response program, clock program and temperature measurement program.

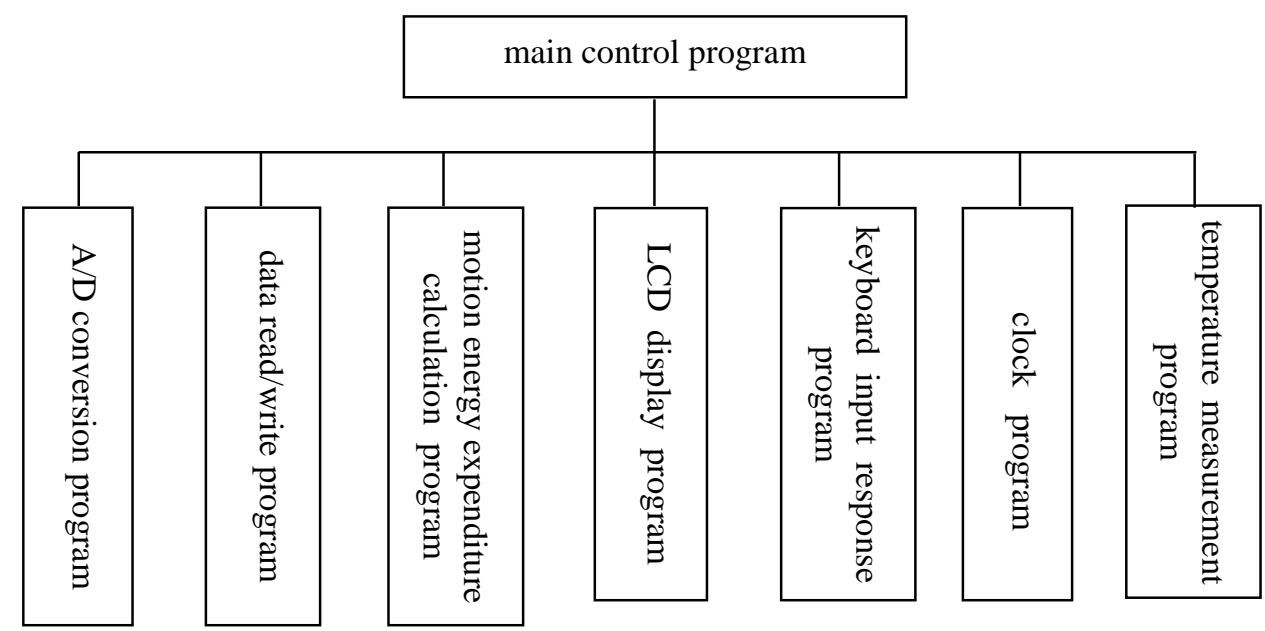

Figure 4. The structure block diagram of software

For further reducing power consumption of the whole system, the software structure was designed to support interrupt program structure and power management by software since MSP430F149 is able to be waken up in 6 microseconds. Specifically, the main control program would turn off hardware circuits or peripherals for power management by its instructions when they were not at work, and also MSP430F149 was set to enter specific low-power mode when it was in idle time.

In A/D conversion program, the A/D hardware module of MSP430F149 was set to work in one-time sequential channel mode. Timer A of MSP430F149 was used to provide timing signal for sampling, and its timing interval was set to be the sampling interval of A/D conversion program. In A/D interrupt service routine, collected data was put into data buffer of MSP430F149.

The temperature from temperature measurement unit and the time from clock unit was read in once per cycle of the main loop of main control program. All data was stored in the data buffer of MSP430F149 temporarily. When the data buffer was full, the collected data would be written into AT45DB321B through page-write mode.

The flowchart of data write program is shown in Fig.5. 


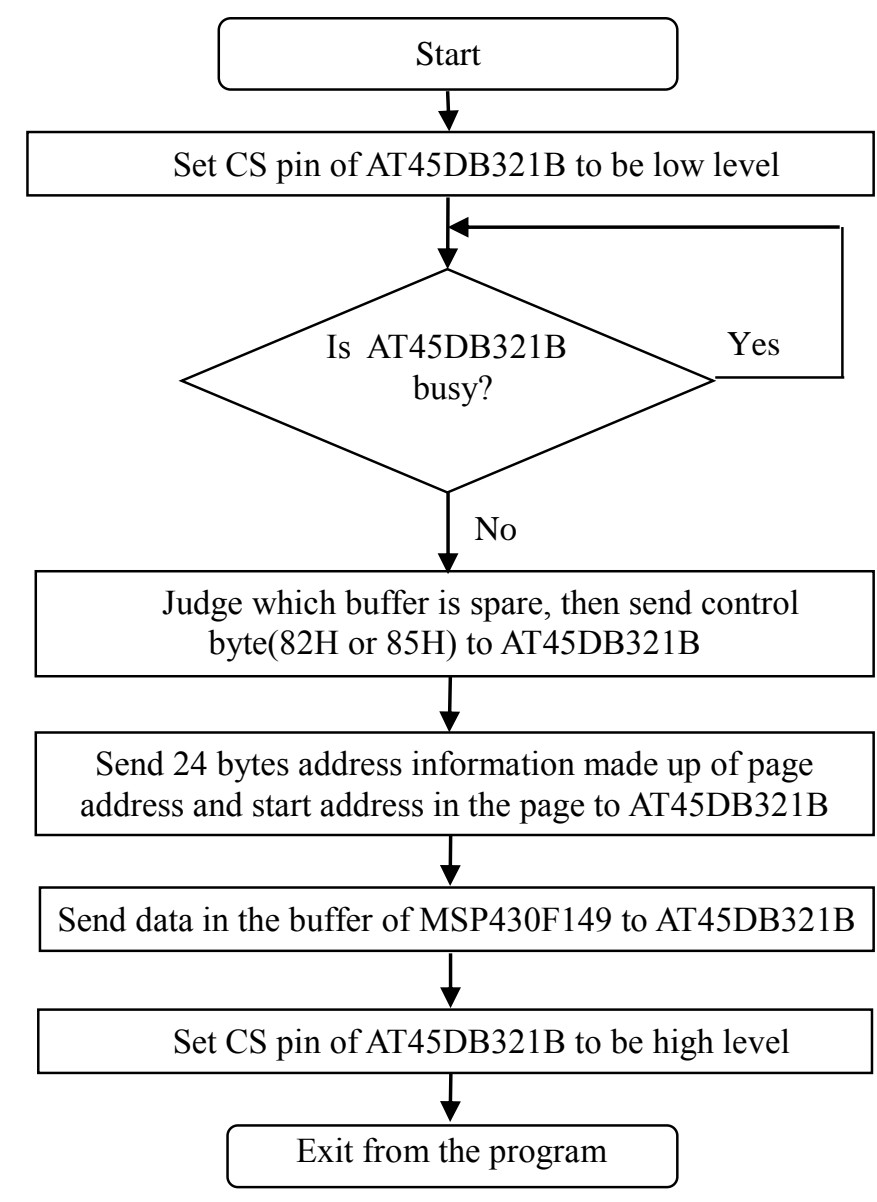

Figure 5. The flowchart of data write program

\section{Conclusions}

In this article, for assessing energy expenditure of physical activity in daily life, a portable measuring instrument based on triaxial accelerometer was presented, in which a combined three-dimensional accelerometer was used to detect frequency and intensity of ambulatory movements. Although it is widely accepted that accelerometers are preferable to evaluate physical activity, for further improving accuracy and validity of measuring method based on accelerometer, classification algorithm of movement type and evaluation algorithm that is suitable for various intensity of ambulatory movements should be greatly improved.

\section{Acknowledgment}

This work was supported by scientific research project of Xi' an Peihua University (PHKT17017).

\section{References}

[1] C.V.C. Bouten, K.T.M. Koekkoek, M. Verduin. 1997. A triaxial accelerometer and portable data processing unit for the assessment of daily physical activity, IEEE Trans. Biomed. Eng., 44: 136-147.

[2] Troiano, Richard P., Berrigan, DAVID. 2008. Physical Activity in the United States Measured by Accelerometer, Medicine \& Science in Sports \& Exercise, 40: 181-188.

[3] Klippel NJ, Heil DP. 2003. Validation of energy expenditure prediction algorithms in adults using the Actical electronic activity monitor, Med Sci Sports Exerc, 35: S284.

[4] Meredith A. Perry, Paul A. Hendrick, et al. 2010. Utility of the RT3 triaxial accelerometer in free living: An investigation of adherence and data loss, Applied Ergonomics, 41: 469-476. 
[5] Juliette Hussey, Kathleen Bennett, et al. 2009. Validation of the RT3 in the measurement of physical activity in children, Journal of Science and Medicine in Sport, 12: 130-133.

[6] Tanaka, Hidenori; Kawamata, Tomoyuki; Gen-No, Hirokazu; Nose, Hiroshi; Kawamata, Mikito. 2015. Evaluation of the Physical Activity of Anesthesiologists in the Operating Room During Daily Work Using a Triaxial Accelerometer, Archives of Environmental \& Occupational Health, Vol.70: 77-80.

[7] ROWLANDS, ALEX V.; YATES, THOMAS; OLDS, TIM S.; DAVIES, ELANIE; KHUNTI, KAMLESH; EDWARDSON, CHARLOTTE L. 2016. Sedentary Sphere: Wrist-Worn Accelerometer-Brand Independent Posture Classification, Medicine \& Science in Sports \& Exercise, Vol.48: 748-754.

[8] Daniel B. Bornstein, Michael W. Beets, et al. 2011. Equating accelerometer estimates of moderate-to-vigorous physical activity: In search of the Rosetta Stone, Journal of Science and Medicine in Sport, 14: 404-410. 